\title{
Electromagnetic force density in electrically and magnetically polarizable media
}

\author{
Iñigo Liberal, ${ }^{1}$ Iñigo Ederra, ${ }^{1}$ Ramón Gonzalo, ${ }^{1}$ and Richard W. Ziolkowski ${ }^{2}$ \\ ${ }^{1}$ Electrical and Electronic Engineering Department, Universidad Pública de Navarra, Campus Arrosadía, 31006 Pamplona, Spain \\ ${ }^{2}$ Department of Electrical and Computer Engineering, University of Arizona, Tucson, Arizona 85721, USA
}

(Received 30 August 2013; published 7 November 2013)

\begin{abstract}
The force density induced by electromagnetic fields in electrically and magnetically polarizable media is studied analytically. Different formulations of the force density as a function of field-related quantities, including the spatial derivatives of the fields, gradients of the field intensity, phase gradients, electromagnetic power flow (Poynting vector field), and kinetic momentum flow, are introduced. These formulations retain certain symmetries with respect to the force expressions introduced in previous works for an isolated particle but also point out fundamental differences, such as the suppression of recoil forces, negative radiation pressure, and far-field gradient forces. It is shown how these analytical formulations also provide the necessary means to elucidate the sign of the force density in complex media and how they can assist the design of sources to manipulate clouds of particles. The theory is illustrated with numerical examples of an insulated Hertzian dipole immersed in different media, including lossy dielectrics, media with negative permittivity and permeability, and zero-index media.
\end{abstract}

DOI: 10.1103/PhysRevA.88.053808

PACS number(s): 42.50.Wk, 41.20.-q, 37.10.Vz

\section{INTRODUCTION}

Mechanical forces induced by electromagnetic fields are leading to unprecedented progress in the development of instrumentation for a wide range of research disciplines, including biology [1,2], chemistry [3], nanotechnology [4], and atomic physics [5]. In essence, a mechanical force arises from the exchange of momentum between the electromagnetic fields and matter [6]. Such forces can also be fundamentally understood as a manifestation of the Lorentz force, and it can be numerically computed through the Maxwell stress tensor [7]. Despite this fundamental perspective, there has been much interest, particularly when it comes to small particles, in finding additional analytical formulations to determine the force exerted on a given object [8-18]. These formulations express the force in terms of field-related quantities, including the spatial derivatives of the local field $[8,9]$, gradients of the field intensity [10], phase gradients [12,13], electromagnetic power (Poynting vector field), and/or kinetic momentum flows [14], as well as the orbital and spin [16-18] components of the electromagnetic kinetic momentum. The interest in these additional formulations lies in the need for analytical tools that can provide physical insight and intuitive understanding in order to boost innovative engineering concepts in the form of novel manipulation and trapping mechanisms.

When a particle or an electrically small inclusion (whose response can be approximated as that of a dipole particle) is not an independent entity but one of the many members of a cloud of particles, i.e., an arbitrary ensemble of particles forming a metamaterial (i.e., an artificial electromagnetic material composed of an ensemble of inclusions) or a natural solid, liquid, or gaseous medium, the force exerted on this entity is significantly different from that exerted on it in isolation. This effect is due to the interaction between the particle of interest and the various other particles composing the cloud. Therefore, to compute the force acting on each single particle, one must first solve a multiple-scattering problem. Unfortunately, this is, in general, not possible due to either computational (e.g., a too large number of particles) or fundamental (e.g., unknown location of the particles) reasons. This complexity of the scattering problem is usually overcome with approximate effective-medium theories [19] that, within the range of validity of the homogenization approach, provide a reasonably accurate (but significantly simpler) solution to the electromagnetic response of the ensemble of particles. Similarly, it is also convenient to predict the forces exerted on the particles constituting the cloud based on the same effective-medium theories. This is indeed possible, and as suggested previously [20], the force exerted on each of the "atoms" composing this effective medium can be simply estimated as $f / N$, where $f$ is the force density exerted on the effective medium and $N$ is the number of atoms per unit volume.

A closed form of the force density $f$ exerted on electrically and magnetically polarizable media was presented by Chu, Haus, and Penfield [21,22] and Einstein and Laub [23,24], which has been recently revisited by Mansuripur $e t$ al. [25,26]. The popular analysis of Gordon [20] for dielectric media leads to a particular version of the same force-density expression. This formalism will be the starting point of the derivations presented in this work. In contrast to the numerous works that discuss the force acting on a single isolated particle in terms of a variety of field-related quantities [8-18], all of the aforementioned references [20-26] study the force density based on the same expression, which emphasizes the action of the electromagnetic field on the electric and magnetic polarization densities of the cloud. Therefore, the first goal of this article is to derive in Sec. II alternative formulations of the force density. While these formulations yield expressions having a certain degree of similarity with respect to those associated with isolated particles, it will be emphasized in Sec. III that there are also fundamental differences that arise from the interaction with neighboring particles. Based on these theoretical considerations, Sec. IV focuses on the force density in the far field of a localized source. As will be shown, this is a nontrivial problem for 
complex media (e.g., a medium with negative permittivity and permeability). To illustrate its impact, the presented theory is illustrated in Sec. V with numerical examples in which an insulated Hertzian dipole is immersed in different media. They will exemplify how the presented formulations serve to elucidate in a straightforward manner the forces produced by such a localized, simple source in a complex medium.

\section{FORCE-DENSITY FORMULATIONS}

According to the formalisms in Refs. [21-26], the force density exerted by an electromagnetic field $(\mathcal{E}, \mathcal{H})$ on an electrically and magnetically polarizable medium (characterized by the electric and magnetic polarization densities, $\mathcal{P}$ and $\mathcal{M}$, respectively, and at most moving at nonrelativistic speeds) is given by

$$
\begin{aligned}
\mathbf{f}(\mathbf{r}, t)= & (\mathcal{P} \cdot \nabla) \mathcal{E}+\partial_{t} \mathcal{P} \times \mu_{0} \mathcal{H} \\
& +(\mathcal{M} \cdot \nabla) \mathcal{H}-\partial_{t} \mathcal{M} \times \varepsilon_{0} \mathcal{E},
\end{aligned}
$$

where $\varepsilon_{0}$ and $\mu_{0}$ are, respectively, the permittivity and permeability of free space. With the $e^{j \omega t}$ convention, the time-harmonic form of the electromagnetic field can be written as

$$
\begin{aligned}
\mathcal{E}(\mathbf{r}, t) & =\operatorname{Re}\left[\mathbf{E}(\mathbf{r}) e^{j \omega t}\right], \\
\mathcal{H}(\mathbf{r}, t) & =\operatorname{Re}\left[\mathbf{H}(\mathbf{r}) e^{j \omega t}\right] .
\end{aligned}
$$

In linear homogeneous isotropic media, characterized by the electric and magnetic susceptibilities, $\chi_{E}=\chi_{E}^{\prime}-j \chi_{E}^{\prime \prime}$ and $\chi_{H}=\chi_{H}^{\prime}-j \chi_{H}^{\prime \prime}$, where the loss terms $\chi_{E}^{\prime \prime}, \chi_{H}^{\prime \prime}>0$ for passive media, the time-harmonic polarization densities take the forms

$$
\begin{gathered}
\mathcal{P}(\mathbf{r}, t)=\operatorname{Re}\left[\varepsilon_{0} \chi_{E} \mathbf{E}(\mathbf{r}) e^{j \omega t}\right], \\
\mathcal{M}(\mathbf{r}, t)=\operatorname{Re}\left[\mu_{0} \chi_{H} \mathbf{H}(\mathbf{r}) e^{j \omega t}\right] .
\end{gathered}
$$

As illustrated by (4) and (5), local electric and magnetic susceptibilities are assumed to be valid descriptions of the medium. Therefore, when it consists of an ensemble of particles that form an artificial electromagnetic material, both the particle size and the separation between particles must be much smaller than the operational wavelength in the medium. In fact, it has been recognized that nonlocal effects appear as the particle size and the interparticle distances increase [27-33]. The study of the force density in the presence of larger particles and the associated complex nonlocal effects is left for future efforts.

Under these circumstances, the time averaged force density corresponding to (1) can be written as

$$
\begin{aligned}
\langle\mathbf{f}\rangle= & \frac{1}{2} \operatorname{Re}\left\{\varepsilon_{0} \chi_{E}(\mathbf{E} \cdot \nabla) \mathbf{E}^{*}+j \omega \mu_{0} \varepsilon_{0} \chi_{E} \mathbf{E} \times \mathbf{H}^{*}\right. \\
& \left.+\mu_{0} \chi_{H}(\mathbf{H} \cdot \nabla) \mathbf{H}^{*}-j \omega \mu_{0} \varepsilon_{0} \chi_{H} \mathbf{H} \times \mathbf{E}^{*}\right\} .
\end{aligned}
$$

While this formulation can be applied to compute the force and acceleration produced in a background medium due to the presence of an electromagnetic field, we introduce now alternative formulations of the force density. In this manner, we will be able to relate the different force components to field-related quantities that can be engineered by designing the characteristics of a source to achieve a particular manipulation system.

To begin with, (6) can be written in a much more compact form by introducing the Maxwell curl equations

$$
\begin{gathered}
\nabla \times \mathbf{E}=-j \omega\left(\mu_{0} \mathbf{H}+\mathbf{M}\right), \\
\nabla \times \mathbf{H}=j \omega\left(\varepsilon_{0} \mathbf{E}+\mathbf{P}\right)
\end{gathered}
$$

into (6) and noting the vector calculus identity [34]

$$
\mathbf{A} \cdot(\boldsymbol{\nabla}) \mathbf{A}^{*}=(\mathbf{A} \cdot \nabla) \mathbf{A}^{*}+\mathbf{A} \times\left(\boldsymbol{\nabla} \times \mathbf{A}^{*}\right),
$$

which leads to the expression

$$
\langle\mathbf{f}\rangle=\frac{1}{2} \operatorname{Re}\left\{\varepsilon_{0} \chi_{E} \mathbf{E} \cdot(\boldsymbol{\nabla}) \mathbf{E}^{*}+\mu_{0} \chi_{H} \mathbf{H} \cdot(\boldsymbol{\nabla}) \mathbf{H}^{*}\right\} .
$$

Equation (10) relates the force density to the spatial derivatives of the electromagnetic field. It is perhaps the most compact and efficient way to compute the force density. Note that this formulation is analogous to the expressions of the force exerted on an isolated particle discussed in [8,9]. We emphasize that this correspondence is by no means trivial since the Maxwell curl equations (7) and (8) governing the fields in electrically and magnetically polarizable media contain additional terms due to the presence of the polarization currents. Despite this fact, the contributions from those terms cancel out in (6), and the correspondence with the isolated particle expressions is kept.

While (10) represents a compact and efficient way to compute the force density, other formulations are more convenient to emphasize the underlying physics. For example, this force density can be rewritten by emphasizing the reactive (terms proportional to $\chi_{E}^{\prime}, \chi_{H}^{\prime}$ ) and dissipative (terms proportional to $\left.\chi_{E}^{\prime \prime}, \chi_{H}^{\prime \prime}\right)$ components. To this end, note that, without any loss of generality, the electric and magnetic fields can be written as

$$
\begin{aligned}
& \mathbf{E}(\mathbf{r})=\sum_{q}\left|E_{q}(\mathbf{r})\right| e^{-j \Phi_{q}^{E}(\mathbf{r})} \widehat{\mathbf{q}}, \\
& \mathbf{H}(\mathbf{r})=\sum_{q}\left|H_{q}(\mathbf{r})\right| e^{-j \Phi_{q}^{H}(\mathbf{r})} \widehat{\mathbf{q}},
\end{aligned}
$$

where $\left|E_{q}(\mathbf{r})\right|,\left|H_{q}(\mathbf{r})\right|$ and $\Phi_{q}^{E}(\mathbf{r}), \Phi_{q}^{H}(\mathbf{r})$ are the magnitude and phase distributions of the $q$ th polarization component (with unitary vector $\widehat{\mathbf{q}}$ ) for the electric and magnetic fields, respectively. Introducing (11) and (12) into (10) and operating the gradient operator on each of the $q$ th polarization components, it is found that the force density can also be written as

$$
\begin{aligned}
\langle\mathbf{f}\rangle= & \frac{1}{4}\left[\varepsilon_{0} \chi_{E}^{\prime} \nabla|\mathbf{E}|^{2}+\mu_{0} \chi_{H}^{\prime} \nabla|\mathbf{H}|^{2}\right] \\
& +\frac{1}{2} \sum_{q}\left[\varepsilon_{0} \chi_{E}^{\prime \prime}\left|E_{q}\right|^{2} \nabla \Phi_{q}^{E}+\mu_{0} \chi_{H}^{\prime \prime}\left|H_{q}\right|^{2} \nabla \Phi_{q}^{H}\right] .
\end{aligned}
$$

Equation (13) illustrates how the reactive part of the force density is proportional to the gradients of the electric and magnetic field intensities, while the dissipative part of the force density is proportional to the phase gradients of the electric and magnetic fields. This formulation is fully analogous to the description of the forces on an isolated particle presented in $[12,13]$.

Recent works relate the dissipative part of the force exerted on an isolated particle to the orbital part of the kinetic momentum (the electromagnetic energy flow) [16]. To 
illustrate this point, let us define the vector fields $\mathbf{g}_{O}^{E}, \mathbf{g}_{O}^{H}$ (with momentum density units) as

$$
\begin{aligned}
\mathbf{g}_{O}^{E} & =\frac{\varepsilon_{0}}{2 \omega} \operatorname{Im}\left[\mathbf{E} \cdot(\nabla) \mathbf{E}^{*}\right], \\
\mathbf{g}_{O}^{H} & =\frac{\mu_{0}}{2 \omega} \operatorname{Im}\left[\mathbf{H} \cdot(\nabla) \mathbf{H}^{*}\right] .
\end{aligned}
$$

Thus, splitting the terms in the brackets into their real and imaginary parts, the force density (10) can be rewritten as

$$
\langle\mathbf{f}\rangle=\frac{1}{4}\left[\varepsilon_{0} \chi_{E}^{\prime} \nabla|\mathbf{E}|^{2}+\mu_{0} \chi_{H}^{\prime} \nabla|\mathbf{H}|^{2}\right]+\omega\left[\chi_{E}^{\prime \prime} \mathbf{g}_{O}^{E}+\chi_{H}^{\prime \prime} \mathbf{g}_{O}^{H}\right] .
$$

As noted in [16], the vector fields $\mathbf{g}_{O}^{E}$ and $\mathbf{g}_{O}^{H}$ in free space correspond to the orbital part of the kinetic momentum with respect to the electric and magnetic fields, respectively. However, as demonstrated in the Appendix, the vector fields $\mathbf{g}_{O}^{E}$ and $\mathbf{g}_{O}^{H}$ within electrically and magnetically polarizable media do not correspond to either the orbital part of the kinetic (Abraham) momentum, $\mathbf{g}_{\text {kin }}=\frac{\mu_{0} \varepsilon_{0}}{2} \operatorname{Re}\left[\mathbf{E} \times \mathbf{H}^{*}\right]$, or to the orbital part of the canonical (Minkowski) momentum, $\mathbf{g}_{\text {can }}=$ $\frac{\mu_{0} \varepsilon_{0}}{2} \operatorname{Re}\left[\mathbf{D} \times \mathbf{B}^{*}\right]$. On the other hand, $\mathbf{g}_{O}^{E}$ and $\mathbf{g}_{O}^{H}$ are identified with the orbital part of the vector fields $\mathbf{g}_{\text {kin }}+\frac{1}{2} \operatorname{Re}\left\{\varepsilon_{0} \mathbf{E} \times \mathbf{M}^{*}\right\}$ and $\mathbf{g}_{\text {kin }}+\frac{1}{2} \operatorname{Re}\left\{\mathbf{P} \times \mu_{0} \mathbf{H}^{*}\right\}$, respectively. Apparently, while the dissipative part of the force exerted on an isolated particle can be linked to the orbital part of the kinetic momentum, the same decomposition does not hold in principle for the force density in electrically and magnetically polarizable media. As a matter of fact, to the best knowledge of the authors and as mentioned in the Appendix, a suitable decomposition of the kinetic and canonical momentums in lossy media has not been proposed yet. This matter is left for future efforts.

To finalize the formulations, we derive an alternative forcedensity expression that is convenient for the design of sources to manipulate the background cloud, emphasizing one or the other force-component behavior. To this end, we apply the imaginary operator on (9), and then each addend in (9) is evaluated according to the vector calculus identities [35]

$$
\begin{gathered}
\operatorname{Im}\left[(\mathbf{A} \cdot \nabla) \mathbf{A}^{*}\right]=\frac{1}{2 j} \nabla \times\left(\mathbf{A}^{*} \times \mathbf{A}\right), \\
\operatorname{Im}\left[\mathbf{E} \times\left(\nabla \times \mathbf{E}^{*}\right)\right]=-2 \omega\left[\mu^{\prime} \mathbf{S}_{R}-\mu^{\prime \prime} \mathbf{S}_{I}\right], \\
\operatorname{Im}\left[\mathbf{H} \times\left(\nabla \times \mathbf{H}^{*}\right)\right]=-2 \omega\left[\varepsilon^{\prime} \mathbf{S}_{R}+\varepsilon^{\prime \prime} \mathbf{S}_{I}\right],
\end{gathered}
$$

where $\varepsilon=\varepsilon^{\prime}-j \varepsilon^{\prime \prime}=\varepsilon_{0}\left(1+\chi_{E}\right)$ and $\mu=\mu^{\prime}-j \mu^{\prime \prime}=$ $\mu_{0}\left(1+\chi_{H}\right)$ are the medium's permittivity and permeability, respectively, with $\varepsilon^{\prime \prime}, \mu^{\prime \prime}>0$ for passive media. The terms $\mathbf{S}_{R}$ and $\mathbf{S}_{I}$ represent the real and imaginary parts of the complex Poynting vector field $\mathbf{S}_{C}$, given by

$$
\mathbf{S}_{C}=\mathbf{S}_{R}+j \mathbf{S}_{I}=\frac{1}{2} \mathbf{E} \times \mathbf{H}^{*} .
$$

Upon substitution of (17)-(19) into (9) and then (9) into (10), we find that the force density can be rewritten as

$$
\begin{aligned}
\langle\mathbf{f}\rangle= & \frac{\varepsilon^{\prime}-\varepsilon_{0}}{4} \nabla|\mathbf{E}|^{2}+\frac{\mu^{\prime}-\mu_{0}}{4} \nabla|\mathbf{H}|^{2} \\
& +\frac{\varepsilon^{\prime \prime}}{4 j} \nabla \times\left(\mathbf{E}^{*} \times \mathbf{E}\right)+\frac{\mu^{\prime \prime}}{4 j} \nabla \times\left(\mathbf{H}^{*} \times \mathbf{H}\right) \\
& +\omega\left[\varepsilon^{\prime \prime} \mu^{\prime}+\mu^{\prime \prime} \varepsilon^{\prime}\right] \mathbf{S}_{R} .
\end{aligned}
$$

It is apparent that (21) is closely connected to the analyses of the forces acting on a single particle discussed in [14,15,17], where the force is divided into gradient forces (first line), forces associated with nonuniformities in the polarization distribution (second line), and force terms associated with the Poynting vector field (third line), usually labeled as radiation pressure [17], or traditional radiation pressure [16].

We have found that this formulation is particularly convenient to design electromagnetic manipulation systems to control the background media, e.g., gasses, clouds of nanoparticles, etc. This is due to the fact that each force component is associated with a field quantity that can be engineered through the sources of the electromagnetic fields. These tailored sources constitute the manipulation system.

To begin, the gradient (reactive) force components (first line) are dominant for low-loss background media and, in general, within the near field of the sources. Since the gradients of the field intensities decay away from the source region, they can easily be tuned by modifying the source geometry, e.g., the superposition of the fields generated by several individual radiators (i.e., the sources). Moreover, the relative importance of the electric or magnetic field intensities can be tuned by constructing the source as a combination of either electric or magnetic (i.e., constructed as loop structures) dipoles. The addends in the second line are proportional to the spatial inhomogeneities of the polarization of the electric and magnetic fields. They can be tuned by combining dipoles with different orientations and relative phases. It has been found that these force components can be exploited to trap single particles at their resonant frequency [18], and Eq. (21) suggests that similar strategies could be adopted to manipulate the background media. Finally, the addend in the third line is proportional to the power flow of the electromagnetic field. It will always be present in any real media because the loss terms only go to zero for ideal materials. Therefore, it can be controlled by tailoring the radiation power pattern of the sources, which is a common exercise in antenna engineering, e.g., for radar and communications applications.

\section{PECULIARITIES OF THE FORCE DENSITY}

The previous section introduced different formulations [(10), (13), (16), and (21)] of the force density in an electrically and magnetically polarizable medium. All of these formulations are related to previous reports discussing the forces on a single dipolar particle [8-18]. However, while it was expected that there would be a certain degree of similarity between forces exerted on isolated particles and those acting on a medium composed of many dipolar particles, it was also anticipated that there must be fundamental differences as well, which arise from the interaction between the different particles composing the media. This section gathers the main peculiarities of the force density in the cloud in contrast to the force exerted on a single, isolated dipolar particle.

Suppression of the recoil force. As could be expected, the role of the electric and magnetic susceptibilities, $\chi_{E}, \chi_{H}$, in (16) and (21) is very similar to that of the electric and magnetic polarizabilities, $\alpha_{e e}, \alpha_{m m}$, in describing the forces exerted on a dipolar particle. However, an important difference is that while $\alpha_{e e}^{\prime \prime}, \alpha_{m m}^{\prime \prime}$ are nonzero for lossless particles (i.e., 
their values represent scattering losses), $\chi_{E}^{\prime \prime}, \chi_{H}^{\prime \prime}$ are zero for lossless media [27]. The physical process behind this effect is that the interference between the scattering and coupling suppresses the individual scattering losses of the particles within a homogeneous cloud. It gives rise to uniform propagating fields $[19,32]$. In this manner, scattering losses are suppressed, and the proper energy balance is maintained for the effective homogeneous material [19,27,31,32]. These suppression effects can be rigorously proven for a number of regular lattices and random mixtures with uniform distributions (see, e.g., [31,32] for a discussion of scattering loss suppression in periodical structures and [36] for a historical review on scattering loss suppression in gases).

Therefore, the absorption scattering force associated with lossless particles consists of a recoil effect in response to a reradiation process. In essence, the reradiation from the lossless particle modifies the momentum carried by the electromagnetic field through interference, and the particle is subjected to a response force to ensure momentum conservation. By contrast, the fields propagating through a homogeneous medium are uniform, and a lossless medium explicitly has $\chi_{E}^{\prime \prime}, \chi_{H}^{\prime \prime}=0$, and consequently, there are no recoil effects in the force density. Therefore, the force density acting on a lossless medium reduces to the reactive contributions of the electric and magnetic gradient forces.

Despite this fact, it is worth noting that, in practice, random suspensions of particles and gaseous media might feature nonuniformities and fluctuations in the density of particles [36], as well as particle sizes or interparticle distances comparable to the wavelength in the medium [28,29], which give rise to local scattering losses. In those cases, the effectivemedium precepts break down, and $\chi_{E}^{\prime \prime}, \chi_{H}^{\prime \prime}=0$ are nonzero even for media composed of lossless particles. It can be concluded that, in general, the radiation pressure is reduced within a cloud of particles (with respect to the radiation pressure felt by an isolated particle) because of shielding effects and that this reduction gets larger as the cloud becomes more uniform.

Similarly, (21) is missing the term corresponding to the cross-coupling between the electric and magnetic dipole excitations present in the force exerted on a single particle $[14,15]$. As pointed out in [14], this term is associated with interference effects between the electric and magnetic dipole components of the field reradiated by a single particle, which shape the directional properties of the reradiated field. Since all interference phenomena are included in the homogenization process, it should not be surprising that this term does not contribute to the force density in a homogeneous medium.

Negative radiation pressure in passive media. Following the formulation of the forces exerted on single dipolar particles, the force term associated with the Poynting vector field [third line of (21)] can be referred to as the radiation pressure [17], or traditional radiation pressure [16]. For single particles, this force component has been found to be repulsive for passive particles and attractive for active particles, provided that the gain effect is large enough to overcome the absorption and scattering losses [37]. In essence, this force term is directed along the direction of the power flow, multiplied by the sign of the extinction cross section. However, it is apparent from (21) that the radiation pressure term of the force density follows the sign of $\varepsilon^{\prime \prime} \mu^{\prime}+\mu^{\prime \prime} \varepsilon^{\prime}$. According to [38,39], $\operatorname{sgn}\left(\varepsilon^{\prime \prime} \mu^{\prime}+\right.$ $\left.\mu^{\prime \prime} \varepsilon^{\prime}\right)=\operatorname{sgn}\left(k^{\prime}\right)$ in passive media. Therefore, this term is negative in passive media supporting backward waves $k^{\prime}<0$, e.g., a medium having negative permittivity and permeability [38]. Intriguingly, this force component can also be zero for zero-index (ZI) media [40] $\left(\mu^{\prime}, \varepsilon^{\prime}=0\right.$ or $\left.\chi_{E}^{\prime}, \chi_{H}^{\prime}=-1\right)$, even if they are lossy.

Far-field gradient forces. The forces exerted on a single particle located in the far field of a localized source asymptotically reduce to the radiation pressure term decaying as $r^{-2}$ [18]. On the other hand, the fields decay exponentially when propagating through a lossy medium. This effect produces additional gradients of the field intensities that result from the gradient forces which decay as $r^{-2}$. From a microscopic standpoint, a single particle is affected by the radiation pressure of a propagating far-field wave. On the other hand, in lossy media the extinction of the propagating field produced by the neighboring particles contained in a mixture of them results in gradients of the field intensity illuminating the particle, which, in turn, gives rise to the reactive gradient forces even in the far field. This effect is discussed in the following section.

\section{FORCE DENSITY IN THE FAR FIELD}

Apparently, the combination of possible negative radiation pressure and far-field gradient forces could lead to an arbitrary sign of the force density in the far field, even for passive media. This effect would contradict the common belief that as a propagating field (in the sense of a field decoupled from the sources) loses momentum by dissipation, the background medium should feel a force in the direction of the propagation to compensate for this loss of momentum. To clarify this issue, note that, without any loss of generality, the fields produced by a set of sources with finite extent can be written in the $r \rightarrow \infty$ asymptotic limit as [41]

$$
\begin{gathered}
E_{\theta} \approx \frac{e^{-j k r}}{r} f_{\theta}(\phi, \theta), \\
E_{\phi} \approx \frac{e^{-j k r}}{r} f_{\phi}(\phi, \theta), \\
\frac{E_{\theta}}{H_{\phi}} \approx-\frac{E_{\phi}}{H_{\theta}} \approx \eta ;
\end{gathered}
$$

that is, the far fields from a finite source are approximately transverse electromagnetic (TEM). The terms $k=k^{\prime}-j k^{\prime \prime}=$ $\omega \sqrt{\mu \varepsilon}$ and $\eta=\eta^{\prime}-j \eta^{\prime \prime}=\sqrt{\mu / \varepsilon}$ stand for the propagation constant and medium impedance within the background media, while $f_{\theta}(\phi, \theta)$ and $f_{\phi}(\phi, \theta)$ represent functions with voltage units describing the elevation and azimuthal variations of the field, which are defined by the sources of the electromagnetic field. The Poynting vector field can be determined by introducing (22)-(24) into (20), leading to

$$
\mathbf{S}_{R} \approx \eta^{\prime} \frac{e^{-2 k^{\prime \prime} r}}{2|\eta|^{2} r^{2}}\left[\left|f_{\theta}(\phi, \theta)\right|^{2}+\left|f_{\phi}(\phi, \theta)\right|^{2}\right] \widehat{\mathbf{r}}
$$

Moreover, it can be readily checked that the radial components of the vectors $\nabla \times\left(\mathbf{E}^{*} \times \mathbf{E}\right)$ and $\nabla \times\left(\mathbf{H}^{*} \times \mathbf{H}\right)$ are zero, so that there are no radial $r^{-2}$ force terms associated with 
nonuniform distributions of the electromagnetic field polarization. Therefore, the radial force density coming from the second line of (21) is zero in the far field of the sources, and the remaining force density can be written as a combination of gradient and radiation pressure forces, which is asymptotically (keeping only the $r^{-2}$ terms) given by

$$
\begin{aligned}
\widehat{\mathbf{r}} \cdot\langle\mathbf{f}\rangle \approx & \left\{-k^{\prime \prime} \frac{1}{\eta^{\prime}}\left[|\eta|^{2}\left(\varepsilon^{\prime}-\varepsilon_{0}\right)+\left(\mu^{\prime}-\mu_{0}\right)\right]\right. \\
& \left.+\omega\left[\varepsilon^{\prime \prime} \mu^{\prime}+\mu^{\prime \prime} \varepsilon^{\prime}\right]\right\}\left(\widehat{\mathbf{r}} \cdot \mathbf{S}_{R}\right)
\end{aligned}
$$

To determine the sign of the radial component of the force density, we then make use of the identities [38,39]

$$
\begin{gathered}
\left(k^{\prime}\right)^{2}-\left(k^{\prime \prime}\right)^{2}=\omega^{2}\left(\mu^{\prime} \varepsilon^{\prime}-\mu^{\prime \prime} \varepsilon^{\prime \prime}\right), \\
2 k^{\prime} k^{\prime \prime}=\omega^{2}\left(\mu^{\prime} \varepsilon^{\prime \prime}+\mu^{\prime \prime} \varepsilon^{\prime}\right), \\
\eta=\frac{\omega \mu}{k}=\omega \frac{\mu^{\prime} k^{\prime}+\mu^{\prime \prime} k^{\prime \prime}+j\left(\mu^{\prime} k^{\prime \prime}-\mu^{\prime \prime} k^{\prime}\right)}{|k|^{2}}, \\
|\eta|^{2}=\frac{\omega^{2}|\mu|^{2}}{|k|^{2}}
\end{gathered}
$$

to rewrite the radial component of the far-field force density simply as

$$
\widehat{\mathbf{r}} \cdot\langle\mathbf{f}\rangle \approx k^{\prime \prime} \frac{e^{-2 k^{\prime \prime} r}}{2 r^{2}}\left(\varepsilon_{0}+\frac{\mu_{0}}{|\eta|^{2}}\right)\left(\left|f_{\theta}\right|^{2}+\left|f_{\phi}\right|^{2}\right) .
$$

It is apparent from (31) that the sign of the asymptotic expression of the radial component of the force density far away from the sources is that of $k^{\prime \prime}$. Therefore, this force component is repulsive for lossy media and attractive for gain media. Bearing in mind momentum conservation, the force exerted by propagating fields along the direction of propagation is repulsive when the amount of momentum decreases (lossy media) and attractive when the amount of momentum increases (gain media). This result is a generalization of previous work $[42,43]$ which was restricted to plane waves.

To finalize, note that this conclusion is based on the asymptotic limit $r \rightarrow \infty$, i.e., keeping the propagating (farfield) $r^{-2}$ terms only. However, the nonpropagating $r^{-3}$ terms can be dominant for a considerable range of distances in the near field, in which attractive forces would be perfectly possible, due to the contribution of the reactive gradient forces. Since the balance between the $r^{-2}$ and $r^{-3}$ force terms depends on the peculiarities of each specific source and its far-, mid-, and near-field components, this effect will not be analytically treated here. However, this effect will be illustrated next through numerical examples.

\section{EXAMPLE: INSULATED HERTZIAN DIPOLE}

Let us make use of a canonical example to clarify the theory and illustrate how the different formalisms provide a convincing description of the force density produced by a localized source in a variety of background media. The geometry of the example is schematically depicted in

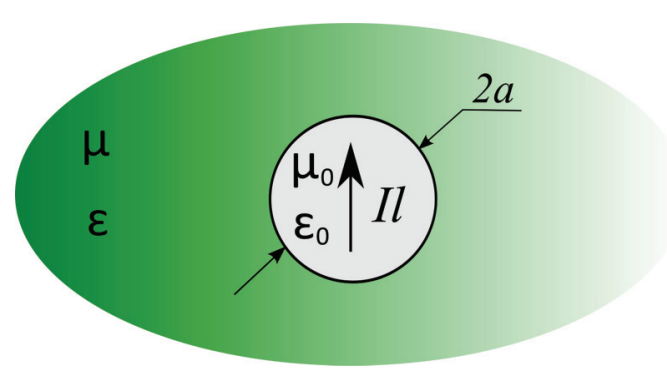

FIG. 1. (Color online) Sketch of the example: an electric Hertzian dipole with current moment $I l$ oriented along $+\widehat{\mathbf{z}}$ is placed at the origin of coordinates within an insulating vacuum sphere of radius $a$ embedded in an unbounded background medium of arbitrary permittivity $\epsilon$ and permeability $\mu$.

Fig. 1: an electric Hertzian dipole with current moment $I l$ is oriented along the $+\widehat{\mathbf{z}}$ direction; it is placed at the origin of the coordinates, and it lies within a sphere of vacuum of radius $a$ embedded in an unbounded background medium of arbitrary permittivity $\epsilon$ and permeability $\mu$. The vacuum sphere insulates the source from the background material. The use of this insulating sphere avoids the singularity that arises from the supplied power when the source region is in contact with a lossy medium [44], so that the force density exerted on the background material can be normalized with respect to the power supplied by the sources, which is the magnitude of interest from an engineering standpoint. In this manner, this canonical example is representative of any small manipulating system consisting of a dipolar antenna (e.g., most state-of-the-art nanoantennas $[45,46]$ ) and serves to illustrate how such localized sources would serve to manipulate the particles which compose the background material surrounding them.

Following a spherical harmonic decomposition (see, e.g., [41]), it can be concluded that in this canonical geometry only the $n=1, m=0$ even TM mode is excited in both the vacuum sphere and the unbounded background medium. Consequently, the fields external to the source region, i.e., the fields where the force density is nonzero, can be written as

$$
\begin{gathered}
\mathbf{E}^{\mathrm{ext}}=E_{0}\left\{2 \frac{\widehat{H}_{1}^{(2)}(k r)}{(k r)^{2}} \cos \theta \widehat{\mathbf{r}}-\frac{\widehat{H}_{1}^{(2)^{\prime}}(k r)}{k r} \sin \theta \widehat{\boldsymbol{\theta}}\right\}, \\
\mathbf{H}^{\mathrm{ext}}=j \frac{E_{0}}{\eta} \frac{\widehat{H}_{1}^{(2)}(k r)}{k r} \sin \theta \widehat{\phi},
\end{gathered}
$$

where $\widehat{H}_{1}^{(2)}(\cdot)$ and $\widehat{H}_{1}^{(2)^{\prime}}(\cdot)$ are, respectively, the Schelkunoff form of the spherical Hankel function of order 1, kind 2 and the derivative with respect to its argument. The constant $E_{0}$ is a constant with electric field units, which is found by solving the boundary value problem on the surface of the vacuum sphere; it is given by

$$
E_{0}=\frac{j \frac{\eta k k_{0}}{4 \pi} I l}{\widehat{H}_{1}^{(2)}(k a) \widehat{J}_{1}^{\prime}\left(k_{0} a\right)-\frac{\eta}{\eta_{0}} \widehat{H}_{1}^{(2)^{\prime}}(k a) \widehat{J}_{1}\left(k_{0} a\right)},
$$

where $\widehat{J_{1}}(\cdot)$ and $\widehat{J_{1}^{\prime}}(\cdot)$ are, respectively, the Schelkunoff form of the spherical Bessel function of order 1, kind 1 and the derivative with respect to its argument. The power supplied by the dipole that is radiated into the external medium can 
be found by calculating the flux of the Poynting vector field through the sphere of radius $r=a$. This supplied power is dissipated in the external medium as the electromagnetic wave propagates away from its source. It is given explicitly as

$$
\begin{aligned}
P_{\text {sup }} & =\frac{1}{2} \oiint_{S_{r=a}} \operatorname{Re}\left[\mathbf{E} \times \mathbf{H}^{*}\right] \cdot \widehat{\mathbf{n}} d S \\
& =\frac{4 \pi}{3}\left|\frac{E_{0}}{k}\right|^{2} \operatorname{Re}\left\{\frac{j}{\eta} \widehat{H}_{1}^{(2)^{\prime}}(k a)\left[\widehat{H}_{1}^{(2)}(k a)\right]^{*}\right\} .
\end{aligned}
$$

\section{A. Dielectric media}

Since there is little to no magnetic activity at frequencies in the millimeter-wave, infrared, and optical domains, the electromagnetic response of most natural mixtures of particles can be associated with that of dielectric materials. In these simple media, the extent of losses determines whether the reactive gradient forces or the dissipative radiation pressure dominates the overall force density. To illustrate this fact, Fig. 2 depicts the color map and quiver plot (arrows) of the force density in the $x z$ plane for three different lossy dielectrics ( $\left.\mu_{r}=1\right)$ with relative permittivities: (a) $\varepsilon_{r}=2.0-j 0.001$, (b) $\varepsilon_{r}=2-j 0.01$, and (c) $\varepsilon_{r}=2.0-j 0.1$. In particular, the color maps represent the magnitude of the force density at each specific location. It is plotted in decibel scale, with respect to the power supplied by the source, and normalized to a reference value of $1(\mathrm{pN} / \mathrm{mW}) / \lambda_{0}^{3}$, with $\lambda_{0}$ being the free-space operational wavelength. The volume covered by the source-vacuum sphere region has been indicated with a gray patch. Note that, due to the perfect azimuthal symmetry of the problem, the plot in the $x z$ plane already contains all of the information of the three-dimensional (3D) problem. The source current moment is set to $I l=10^{-3} \lambda_{0} \mathrm{~A} \mathrm{~m}$, and the vacuum sphere has the radius $a=0.1 \lambda_{0}$.

As could be expected from (21), the force density for small losses is dominated by its reactive component, which is associated with the gradient of the electric field intensity. In this manner, the force density is found to be attractive in Figs. 2(a) and 2(b). This is readily apparent from the expression for the radial component of the force density in a lossless dielectric:

$$
\begin{aligned}
\widehat{\mathbf{r}} \cdot\langle\mathbf{f}\rangle= & -k \frac{\varepsilon^{\prime}-\varepsilon_{0}}{2}\left|E_{0}\right|^{2} \\
& \times\left[\frac{\sin ^{2} \theta}{(k r)^{3}}+2 \frac{5 \cos ^{2} \theta-1}{(k r)^{5}}+3 \frac{3 \cos ^{2} \theta+1}{(k r)^{7}}\right] .
\end{aligned}
$$

On the other hand, the dissipative part of the force density should play a major role as the losses increase. Moreover, this effect will become more dramatic as the distance of separation from the sources increases. In fact, as pointed out in Sect. IV, the force density produced by a localized source asymptotically $(r \rightarrow \infty)$ tends to be repulsive. This is evidenced in the $r \ll 1$ and $r \gg 1$ asymptotic limits of the radial component of the force density in a lossy dielectric:

$$
\begin{aligned}
& \widehat{\mathbf{r}} \cdot\langle\mathbf{f}\rangle \approx k^{\prime \prime} \frac{|\eta|^{2} \varepsilon_{0}+\mu_{0}}{2|\eta|^{2}}\left|E_{0}\right|^{2} \frac{\sin ^{2} \theta}{(|k| r)^{2}} e^{-2 k^{\prime \prime} r} \quad \text { for } \quad r \gg 1, \\
& \widehat{\mathbf{r}} \cdot\langle\mathbf{f}\rangle \approx-\frac{3}{2}|k|\left(\varepsilon^{\prime}-\varepsilon_{0}\right)\left|E_{0}\right|^{2} \frac{3 \cos ^{2} \theta+1}{(|k| r)^{7}} e^{-2 k^{\prime \prime} r} \quad \text { for } \quad r \ll 1 .
\end{aligned}
$$

For the sake of brevity, we have omitted the complete force-density expression containing all of the intermediate terms. However, the limiting cases contained in (37) emphasize how, in the presence of losses, the force density is attractive in the vicinity of the source, but it becomes repulsive as the distance increases. This effect is further illustrated by the case shown in Fig. 2(c). While the losses are still relatively small, they are orders of magnitude larger than the other two cases. Consequently, it can be appreciated how the force becomes repulsive as the separation from the source increases.

However, it was also pointed out that due to the suppression of any recoil forces and the presence of far-field gradient forces, the force density can be attractive for a large range of distances. This effect is also illustrated by the numerical results presented in Fig. 2. In particular, even for losses as high as $\varepsilon^{\prime \prime} / \varepsilon^{\prime}=0.05$, the gradient force remains dominant around the source for distances up to 1.6 free-space

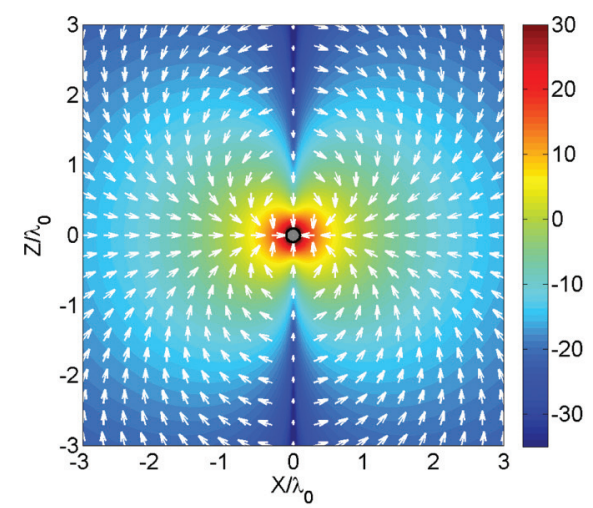

(a)

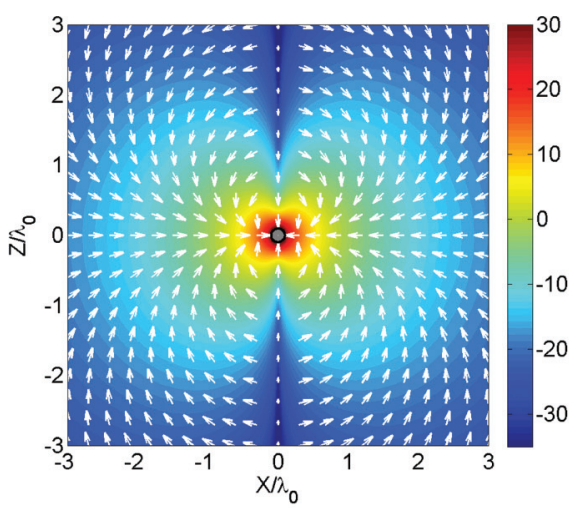

(b)

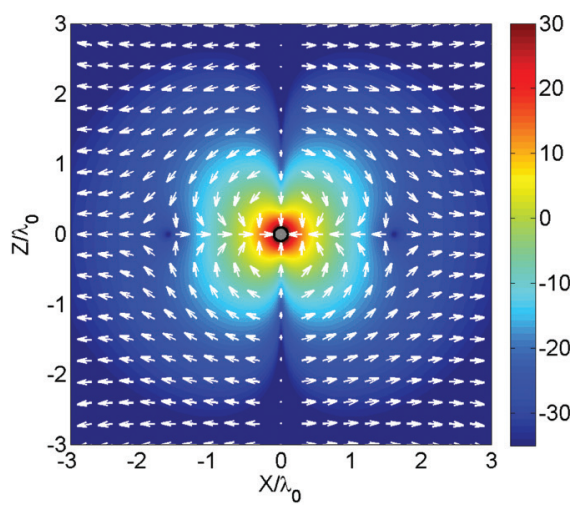

(c)

FIG. 2. (Color online) Color map and quiver plot (arrows) of the force density in the $x z$ plane produced by an electric Hertzian dipole with current moment $I l=10^{-3} \lambda_{0} \mathrm{~A}$ m and oriented along $+\widehat{\mathbf{z}}$ that is placed within a vacuum sphere with radius $a=0.1 \lambda_{0}$, which is embedded within an unbounded lossy dielectric $\left(\mu_{r}=1\right)$ with permittivity: (a) $\varepsilon_{r}=2.0-j 0.001$, (b) $\varepsilon_{r}=2.0-j 0.01$, and (c) $\varepsilon_{r}=2.0-j 0.1$. 
wavelengths [see Fig. 2(c)]. This result suggests that the gradient forces produced by localized sources can be exploited to compress dielectric media composed of loosely bound dipolar particles, e.g., a gaseous medium, even for high losses.

\section{B. Media with negative permittivity and permeability}

It is apparent from (21), in radical opposition to conventional dielectric media, that the force density in media with negative permittivity and permeability is composed of repulsive gradient forces, as well as an attractive radiation pressure. Bearing this in mind and considering also the numerical example presented in Fig. 2(c), it could be argued that the force on a large loss medium with negative permittivity and permeability is attractive at distances arbitrarily far from the sources, where the radiation pressure appears to be dominant. However, as cautioned in Sec. IV, this is not the case. In media with negative permittivity and permeability, the gradient forces become larger than the radiation pressure force even for large losses. Thus, the far-field forces of a localized source are repulsive even for these complex media.

To further illustrate this behavior, Fig. 3 depicts the color map and quiver plot (arrows) of the force density in media characterized by $\varepsilon_{r}, \mu_{r}=-1.0$ and different degrees of the losses. It can be concluded that the force density is repulsive and dominated by the gradient force component, no matter how large the losses are. A similar conclusion can be drawn by inspecting the $r \ll 1$ and $r \gg 1$ asymptotic limits of the radial component of the force density within lossy media characterized by $\varepsilon_{r}^{\prime}, \mu_{r}^{\prime}=-1.0$, which are

$$
\begin{aligned}
& \widehat{\mathbf{r}} \cdot\langle\mathbf{f}\rangle \approx k^{\prime \prime} \varepsilon_{0}\left|E_{0}\right|^{2} \frac{\sin ^{2} \theta}{(|k| r)^{2}} e^{-2 k^{\prime \prime} r} \quad \text { for } \quad r \gg 1, \\
& \widehat{\mathbf{r}} \cdot\langle\mathbf{f}\rangle \approx 3|k| \varepsilon_{0}\left|E_{0}\right|^{2} \frac{3 \cos ^{2} \theta+1}{(|k| r)^{7}} e^{-2 k^{\prime \prime} r} \quad \text { for } \quad r \ll 1 .
\end{aligned}
$$

Therefore, the numerical example in Fig. 3 and the analytical limits in (38) suggest that the particles comprising an artificial material with negative permittivity and permeability would tend to be repelled from a source embedded within them, no matter what the loss amount is or what the distance from the source to a particle is.

\section{Zero-index media}

Equation (21) also identifies ZI media as one of the most intriguing cases. Let us consider a lossy ZI medium with $\varepsilon_{r}, \mu_{r}=-j \delta$. Consequently, $\eta=\eta_{0}$ and $k=-j \delta k_{0}$. Since $k^{\prime}=0$, there is no radiation pressure. Moreover, for low losses $\delta \rightarrow 0$, the field components can be asymptotically written as

$$
\begin{gathered}
E_{r}^{\mathrm{ext}}=2 \frac{E_{\mathrm{ZI}}}{k_{0}^{3}}\left[\frac{\delta k_{0}}{r^{2}}+\frac{1}{r^{3}}\right] e^{-\delta k_{0} r} \cos \theta, \\
E_{\theta}^{\mathrm{ext}}=\frac{E_{\mathrm{ZI}}}{k_{0}^{3}}\left[\frac{\left(\delta k_{0}\right)^{2}}{r}+\frac{\delta k_{0}}{r^{2}}+\frac{1}{r^{3}}\right] e^{-\delta k_{0} r} \sin \theta, \\
H_{\phi}^{\mathrm{ext}}=\frac{E_{\mathrm{ZI}}}{\eta_{0} k_{0}^{3}}\left[\frac{\left(\delta k_{0}\right)^{2}}{r}+\frac{\delta k_{0}}{r^{2}}\right] e^{-\delta k_{0} r} \sin \theta,
\end{gathered}
$$

where

$$
E_{\mathrm{ZI}}=j \frac{\eta_{0} I l}{4 \pi} \frac{k_{0}^{4} a^{2}}{\widehat{J_{1}}\left(k_{0} a\right)} .
$$

It is apparent from (39)-(41) that there is no phase shift between the different components of the electric and magnetic fields. Therefore, $\mathbf{E}^{*} \times \mathbf{E}=0$, and hence, there is no contribution to the force density associated with inhomogeneities in the distribution of the polarization. Consequently, the force density produced by this source on ZI media, although lossy, is uniquely due to the gradient force components. For instance, one finds from (21) that the radial component of the force takes the limiting forms

$$
\begin{aligned}
& \widehat{\mathbf{r}} \cdot\langle\mathbf{f}\rangle \approx \delta^{5} k_{0} \varepsilon_{0}\left|E_{\mathrm{ZI}}\right|^{2} \frac{\sin ^{2} \theta}{\left(k_{0} r\right)^{2}} e^{-2 \delta k_{0} r} \quad \text { for } \quad r \gg 1, \\
& \widehat{\mathbf{r}} \cdot\langle\mathbf{f}\rangle \approx \frac{3}{2} k_{0} \varepsilon_{0}\left|E_{\mathrm{ZI}}\right|^{2} \frac{3 \cos ^{2} \theta+1}{\left(k_{0} r\right)^{7}} e^{-2 \delta k_{0} r} \quad \text { for } \quad r \ll 1 .
\end{aligned}
$$

Moreover, because of the sign of the electric and magnetic polarizabilities, a repulsive force results for all distances. This is evidenced by the approximate radial component result (43) and the entire force field in Fig. 4, which depicts the color map and quiver plot (arrows) of the force density for unbounded

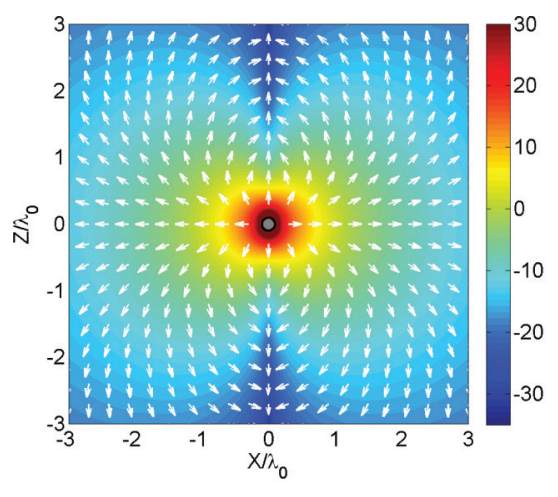

(a)

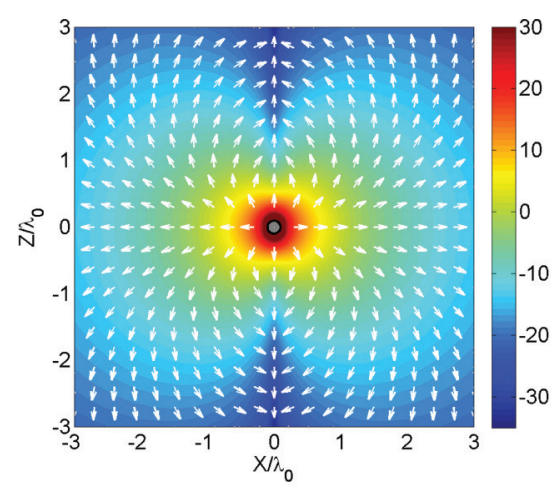

(b)

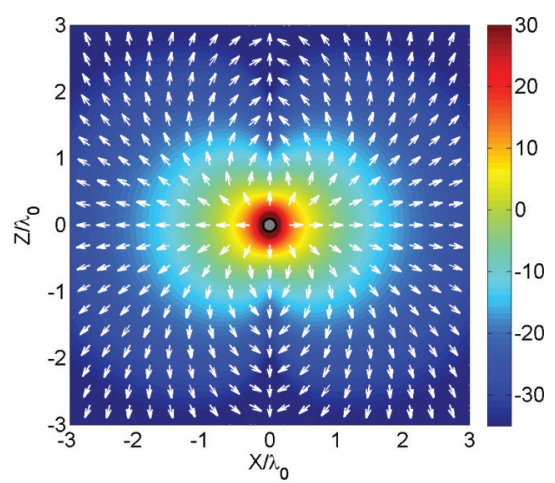

(c)

FIG. 3. (Color online) Color map and quiver plot (arrows) of the force density in the $x z$ plane produced by an electric Hertzian dipole with current moment $I l=10^{-3} \lambda_{0} \mathrm{~A} \mathrm{~m}$ and oriented along $+\widehat{\mathbf{z}}$ that is placed within a vacuum sphere with radius $a=0.1 \lambda_{0}$, which is embedded within an unbounded matched medium characterized by (a) $\varepsilon_{r}, \mu_{r}=-1.0-j 0.001$, (b) $\varepsilon_{r}, \mu_{r}=-1.0-j 0.01$, and (c) $\varepsilon_{r}, \mu_{r}=-1.0-j 0.1$. 


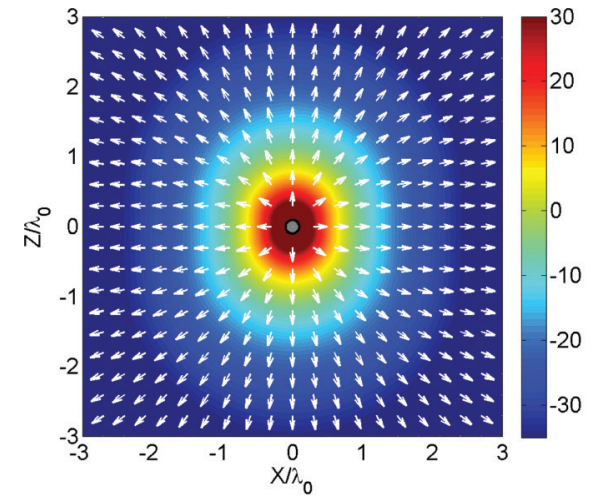

(a)

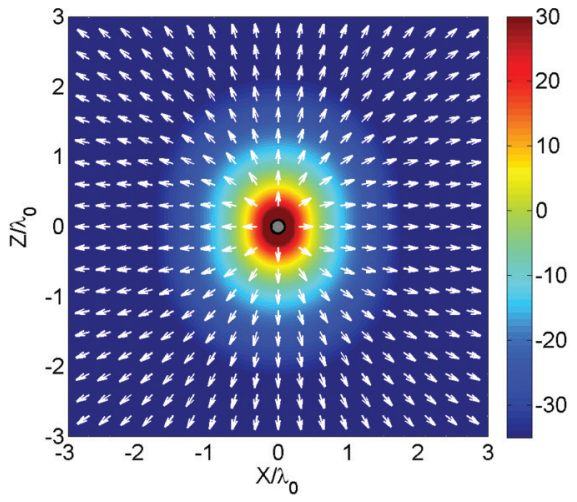

(b)

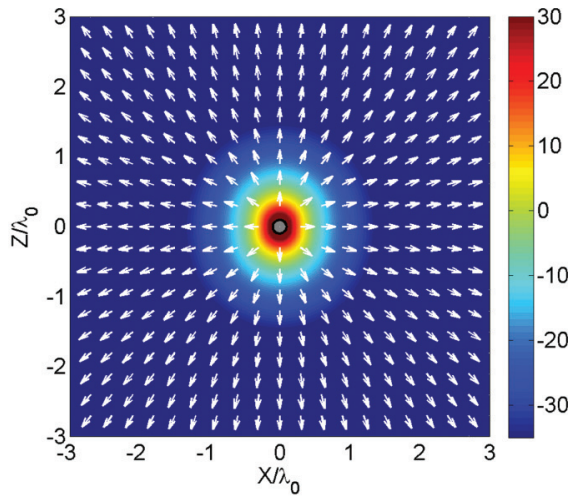

(c)

FIG. 4. (Color online) Color map and quiver plot (arrows) of the force density in the $x z$ plane produced by an electric Hertzian dipole with current moment $I l=10^{-3} \lambda_{0}$ A m and oriented along $+\widehat{\mathbf{z}}$ that is placed within a vacuum sphere with radius $a=0.1 \lambda_{0}$, which is embedded within an unbounded matched medium characterized by (a) $\varepsilon_{r}, \mu_{r}=-j 0.001$, (b) $\varepsilon_{r}, \mu_{r}=-j 0.01$, and (c) $\varepsilon_{r}, \mu_{r}=-j 0.1$.

ZI media with various losses. In addition, Fig. 4 reveals how ZI media emphasize the reactive components of the source fields. For example, the color maps of the force density are characterized by ellipsoidal contours rather than the $\sin ^{2} \theta$ patterns of previous examples (see Figs. 2 and 3). Furthermore, the enhancement on the reactive fields results in a larger force density per watt of supplied power than in the previous examples, even though the dominant force term decays faster as $r^{-7}$. Therefore, this numerical example suggests that the particles composing an artificial material with ZI properties are repelled from localized sources placed within such artificial media and that the forces in the vicinity of the sources are enhanced by the ZI behavior since the near-field components of the source field are incremented due to the influence of the surrounding medium.

To clarify this behavior further, let us theoretically evaluate the exact $\delta=0$ limit, i.e., consider an ideal, lossless ZI medium. It can be concluded from (39)-(41) that only the $r^{-3}$ term of the electric field is nonvanishing. In addition, the magnetic field goes to zero, so that the fields can be simply written as

$$
\begin{gathered}
\mathbf{E}^{\mathrm{ext}}=j \frac{\eta_{0} I l}{4 \pi} \frac{k_{0} a^{2}}{\widehat{J}_{1}\left(k_{0} a\right)} \frac{2 \cos \theta \widehat{\mathbf{r}}+\sin \theta \widehat{\boldsymbol{\theta}}}{r^{3}}, \\
\mathbf{H}^{\mathrm{ext}}=0,
\end{gathered}
$$

and the radial component of the force density becomes

$$
\widehat{\mathbf{r}} \cdot\langle\mathbf{f}\rangle \approx \frac{3}{2} \varepsilon_{0}\left|\frac{\eta_{0} I l}{4 \pi} \frac{k_{0} a^{2}}{\hat{J}_{1}\left(k_{0} a\right)}\right|^{2} \frac{3 \cos ^{2} \theta+1}{r^{7}} .
$$

It is apparent from (44) and (45) that the field excited within an ideal lossless ZI medium would be given by the combination of a quasistatic electric field and a null magnetic field. This solution is consistent with the appreciation that, within a lossless ZI medium, the electric field must be a curl-free quantity, while the magnetic field reduces to a quasistatic solution [40]. It is also consistent with the concept that in a zero-index medium, the effective wavelength is infinite (i.e., the index of refraction is zero) and the static electric dipole field is recovered. In this example there are no electric currents imprinted on the ZI media, which results in a zero magnetic field. Thus, the force density in this ideal medium would be reduced simply to the gradient force component associated with the intensity of the electric field.

\section{CONCLUSIONS}

The force density induced by electromagnetic fields in electrically and magnetically polarizable media was investigated analytically. Starting from the Chu-Haus-Penfield and/or Einstein-Laub formalism, we have derived different expressions of the force density that describe it in terms of field-related quantities, including the spatial derivatives of the fields, gradients of the field intensity, phase gradients, electromagnetic power and kinetic momentum flows, and nonuniformities in the polarization distribution. While these formulations are analogous in many ways to the force exerted on a single dipolar particle, we have also identified fundamental differences when a cloud of particles exists. These include the suppression of the recoil forces, negative radiation pressure, and far-field gradient forces. We believe that these force expressions will help in the design of sources to manipulate gasses and clouds of particles which constitute, for example, electromagnetic metamaterials (artificial materials).

These formulations also help to extricate the sign of the force density in complex media. Particular attention has been devoted to the sign of the force density produced by the farfield asymptotic limit of the fields produced by a localized source. While the sign of the force density is nontrivial for complex media (e.g., media with negative permittivity and permeability), it has been demonstrated, in this asymptotic limit, that the force density is repulsive for passive media and attractive for gain media, no matter what the other properties of the media are.

The theory has been clarified making use of a canonical example consisting of an insulated Hertzian dipole immersed in different background media. The cases included lossy dielectrics, media with negative permittivity and permeability, and zero-index media; different levels of the losses were considered in each case. The formulations presented in this work provide a straightforward explanation of the forcedensity behaviors for all these examples. 


\section{APPENDIX: DECOMPOSITION OF THE KINETIC MOMENTUM INTO ORBITAL AND SPIN PARTS}

Consider the kinetic (Abraham) momentum density of the electromagnetic field [6]

$$
\mathbf{g}_{\text {kin }}=\frac{\mu_{0} \varepsilon_{0}}{2} \operatorname{Re}\left[\mathbf{E} \times \mathbf{H}^{*}\right] \text {. }
$$

Substituting $\mathbf{H}=\nabla \times \mathbf{E} /\left(-j \omega \mu_{0}\right)-\mathbf{M} / \mu_{0}$ into (A1) and making use of the vector calculus identities [35]

$$
\begin{gathered}
\mathbf{A} \times\left(\boldsymbol{\nabla} \times \mathbf{A}^{*}\right)=\mathbf{A} \cdot(\boldsymbol{\nabla}) \mathbf{A}^{*}-(\mathbf{A} \cdot \boldsymbol{\nabla}) \mathbf{A}^{*}, \\
\operatorname{Im}\left[(\mathbf{A} \cdot \boldsymbol{\nabla}) \mathbf{A}^{*}\right]=\frac{1}{2 j} \boldsymbol{\nabla} \times\left(\mathbf{A}^{*} \times \mathbf{A}\right),
\end{gathered}
$$

the kinetic momentum density within electrically and magnetically polarizable media can be decomposed as

$$
\mathbf{g}_{\text {kin }}=\mathbf{g}_{O}^{E}+\mathbf{g}_{S}^{E}-\frac{1}{2} \operatorname{Re}\left\{\varepsilon_{0} \mathbf{E} \times \mathbf{M}^{*}\right\},
$$

with

$$
\begin{aligned}
\mathbf{g}_{O}^{E} & =\frac{\varepsilon_{0}}{2 \omega} \operatorname{Im}\left[\mathbf{E} \cdot(\nabla) \mathbf{E}^{*}\right], \\
\mathbf{g}_{S}^{E} & =\frac{\varepsilon_{0}}{4 j \omega} \nabla \times\left(\mathbf{E}^{*} \times \mathbf{E}\right) .
\end{aligned}
$$

Similarly, substituting $\mathbf{E}=\nabla \times \mathbf{H} /\left(j \omega \varepsilon_{0}\right)+\mathbf{P} / \varepsilon_{0}$ into (A1) and making use of the vector calculus identities (A2) and (A3), the kinetic momentum can be decomposed as

$$
\mathbf{g}_{\text {kin }}=\mathbf{g}_{O}^{H}+\mathbf{g}_{S}^{H}-\frac{1}{2} \operatorname{Re}\left\{\mathbf{P} \times \mu_{0} \mathbf{H}^{*}\right\},
$$

with

$$
\begin{aligned}
\mathbf{g}_{O}^{H} & =\frac{\mu_{0}}{2 \omega} \operatorname{Im}\left[\mathbf{H} \cdot(\boldsymbol{\nabla}) \mathbf{H}^{*}\right], \\
\mathbf{g}_{S}^{H} & =\frac{\varepsilon_{0}}{4 j \omega} \nabla \times\left(\mathbf{H}^{*} \times \mathbf{H}\right) .
\end{aligned}
$$

In free space $(\mathbf{M}, \mathbf{P}=0)$, the kinetic momentum density can be decomposed into orbital and spin parts with respect to the electric and/or magnetic field

$$
\begin{aligned}
\left.\mathbf{g}_{\text {kin }}\right|_{\text {free-space }} & =\mathbf{g}_{O}^{E}+\mathbf{g}_{S}^{E}=\mathbf{g}_{O}^{H}+\mathbf{g}_{S}^{H} \\
& =\frac{\mathbf{g}_{O}^{E}+\mathbf{g}_{O}^{H}}{2}+\frac{\mathbf{g}_{S}^{E}+\mathbf{g}_{S}^{H}}{2} .
\end{aligned}
$$

However, it is apparent from (A4) and (A7) that, within electrically and magnetically polarizable media, the vector field pairs $\mathbf{g}_{O}^{E}, \mathbf{g}_{S}^{E}$ and $\mathbf{g}_{O}^{H}, \mathbf{g}_{S}^{H}$ do not correspond to the orbital and spin parts of the kinetic momentum (or to the orbital and spin parts of the canonical momentum density: $\mathbf{g}_{\text {can }}=$ $\left.\frac{1}{2} \operatorname{Re}\left\{\mathbf{D} \times \mathbf{B}^{*}\right\}\right)$, but rather to the orbital and spin parts of the vector fields $\mathbf{g}_{\text {kin }}+\frac{1}{2} \operatorname{Re}\left\{\varepsilon_{0} \mathbf{E} \times \mathbf{M}^{*}\right\}$ and $\mathbf{g}_{\text {kin }}+\frac{1}{2} \operatorname{Re}\{\mathbf{P} \times$ $\left.\mu_{0} \mathbf{H}^{*}\right\}$, respectively. Intriguingly, as shown in Sec. II, it is the orbital parts of these vector fields that define the dissipative part of the force density.

We also emphasize that, to the best knowledge of the authors, there has not been a decomposition of the kinetic momentum orbital and spin parts within a lossy medium reported. In recent papers $[16,47]$, the decomposition of the kinetic momentum density into orbital parts has been extended to electrically and magnetically polarizable media by defining the alternative vector fields, $\widetilde{\mathbf{g}}_{O}^{E}, \widetilde{\mathbf{g}}_{S}^{E}, \widetilde{\mathbf{g}}_{O}^{H}, \widetilde{\mathbf{g}}_{S}^{H}$, given by

$$
\begin{gathered}
\widetilde{\mathbf{g}}_{O}^{E}=\frac{\varepsilon_{0}}{2 \omega} \operatorname{Im}\left[\frac{\mu_{0}}{\mu} \mathbf{E} \cdot(\nabla) \mathbf{E}^{*}\right], \\
\widetilde{\mathbf{g}}_{S}^{E}=-\frac{\varepsilon_{0}}{2 \omega} \operatorname{Im}\left[\frac{\mu_{0}}{\mu}(\mathbf{E} \cdot \nabla) \mathbf{E}^{*}\right], \\
\widetilde{\mathbf{g}}_{O}^{H}=\frac{\mu_{0}}{2 \omega} \operatorname{Im}\left[\frac{\varepsilon_{0}}{\varepsilon} \mathbf{H} \cdot(\nabla) \mathbf{H}^{*}\right], \\
\widetilde{\mathbf{g}}_{S}^{H}=-\frac{\mu_{0}}{2 \omega} \operatorname{Im}\left[\frac{\varepsilon_{0}}{\varepsilon}(\mathbf{H} \cdot \nabla) \mathbf{H}^{*}\right] .
\end{gathered}
$$

However, while we consider that the decomposition into orbital and spin parts according to the vector fields (A11)-(A14) is well founded for lossless media, we also believe that it loses its physical meaning for lossy media. To illustrate this fact, consider that the permittivity and permeability within a lossy medium take on complex values, and thus, the spin parts are a function of both the real and imaginary parts of the $\left(\mathbf{A}^{*} \cdot \boldsymbol{\nabla}\right) \mathbf{A}$ vector field, where $\mathbf{A}=\mathbf{E}, \mathbf{H}$. Therefore, the spin parts cannot be, in general, written in the form of $\nabla \times\left(\mathbf{A}^{*} \times \mathbf{A}\right)$ vector fields. Thus, they do not correspond to nonuniformities of the electromagnetic field polarization distribution and thus lose their physical meaning.
[1] J. Nilsson, M. Evander, B. Hammarström, and T. Laurell, Anal. Chim. Acta 649, 141 (2009).

[2] J. P. Desai, A. Pillarisetti, and A. D. Brooks, Annu. Rev. Biomed. Eng. 9, 35 (2007).

[3] J. R. Moffitt, Y. R. Chemla, S. B. Smith, and C. Bustamante, Annu. Rev. Biochem. 77, 205 (2008).

[4] M. Dienerowitz, M. Mazilu, and K. Dholakia, J. Nanophoton. 2, 021875 (2008).

[5] T. L. Gustavson, A. P. Chikkatur, A. E. Leanhardt, A. Görlitz, S. Gupta, D. E. Pritchard, and W. Ketterle, Phys. Rev. Lett. 88, 020401 (2001).

[6] S. M. Barnett, Phys. Rev. Lett. 104, 070401 (2010).

[7] J. D. Jackson, Classical Electrodynamics (Wiley, New York, 1999).
[8] P. C. Chaumet and M. Nieto-Vesperinas, Opt. Lett. 25, 1065 (2000)

[9] P. C. Chaumet and M. Nieto-Vesperinas, Phys. Rev. B 61, 14119 (2000).

[10] A. Ashkin, J. M. Dziedzic, J. E. Bjorkholm, and S. Chu, Opt. Lett. 11, 288 (1986).

[11] J. R. Arias-González and M. Nieto-vesperinas, J. Opt. Soc. Am. A 20, 1201 (2003).

[12] Y. Roichman, B. Sun, Y. Roichman, J. Amato-Grill, and D. G. Grier, Phys. Rev. Lett. 100, 013602 (2008).

[13] D. B. Ruffner and D. G. Grier, Phys. Rev. Lett. 111, 059301 (2013).

[14] M. Nieto-Vesperinas, J. J. Sáenz, R. Gómez-Medina, and L. Chantada, Opt. Express 18, 11428 (2010). 
[15] P. C. Chaumet and A. Rahmani, Opt. Express 17, 2224 (2009).

[16] A. Y. Bekshaev, J. Opt. 15, 044004 (2013).

[17] S. Albaladejo, M. Marqués, M. Laroche, and J. J. Sáenz, Phys. Rev. Lett. 102, 113602 (2009).

[18] I. Liberal, I. Ederra, R. Gonzalo, and R. W. Ziolkowski, Phys. Rev. A 87, 063807 (2013).

[19] S. A. Tretyakov, Analytical Modeling in Applied Electromagnetics (Artech House, Boston, 2003).

[20] J. P. Gordon, Phys. Rev. A 8, 14 (1973).

[21] L. Chu, H. Haus, and P. Penfield, Proc. IEEE 54, 920 (1966).

[22] L. J. Chu, H. A. Haus, and P. Penfield, Electrodynamics of Moving Media (MIT Press, Cambridge, MA, 1967).

[23] A. Einstein and J. Laub, Ann. Phys. (Leipzig) 331, 532 (1908).

[24] A. Einstein and J. Laub, Ann. Phys. (Leipzig) 331, 541 (1908).

[25] M. Mansuripur, Phys. Rev. Lett. 108, 193901 (2012).

[26] M. Mansuripur, A. R. Zakharian, and E. M. Wright, Phys. Rev. A 88, 023826 (2013).

[27] A. Alù, Phys. Rev. B 84, 075153 (2011).

[28] C. F. Bohren, J. Atmos. Sci. 43, 468 (1986).

[29] R. G. Barrera and A. García-Valenzuela, J. Opt. Soc. Am. A 20, 296 (2003).

[30] A. Alù, Phys. Rev. B 83, 081102 (2011).

[31] M. G. Silveirinha, Phys. Rev. B 76, 245117 (2007).

[32] P. A. Belov and C. R. Simovski, Phys. Rev. E 72, 026615 (2005).

[33] X. X. Liu, J. W. Massey, M. F. Wu, K. T. Kim, R. A. Shore, A. E. Ylmaz, and A. Alù, Opt. Express 21, 21714 (2013).
[34] R. Feynman, The Feynman Lectures on Physics (AddisonWesley, Boston, 1963), Vol. 2.

[35] This vector calculus identity is not usually present in classic textbooks, but it can be derived as a linear combination of those of [48].

[36] I. I. Sobel'man, Phys. Usp. 45, 75 (2002).

[37] A. Mizrahi and Y. Fainman, Opt. Lett. 35, 3405 (2010).

[38] R. W. Ziolkowski and E. Heyman, Phys. Rev. E 64, 056625 (2001).

[39] S. A. Ramakrishna and O. J. F. Martin, Opt. Lett. 30, 2626 (2005).

[40] R. W. Ziolkowski, Phys. Rev. E 70, 046608 (2004).

[41] R. F. Harrington, Time-Harmonic Electromagnetic Fields (McGraw-Hill, New York, 1961).

[42] K. J. Webb and Shivanand, Phys. Rev. E 84, 057602 (2011).

[43] K. J. Webb and Shivanand, J. Opt. Soc. Am. B 29, 1904 (2012).

[44] C. T. Tai and R. E. Collin, IEEE Trans. Antennas Propag. 48, 1501 (2000).

[45] P. Biagioni, J.-S. Huang, and B. Hecht, Rep. Prog. Phys. 75, 024402 (2012).

[46] A. Alù and N. Engheta, IEEE Trans. Antennas Propag. 61, 1508 (2013).

[47] K. Y. Bliokh and F. Nori, Phys. Rev. A 85, 061801 (2012).

[48] C. A. Balanis, Advanced Engineering Electromagnetics (Wiley, New York, 2012). 